\title{
Redécouverte de Nomada agrestis FABRICIUS (Hymenoptera, Apidae) en France méditerranéenne
}

\author{
Par Nicolas J Vereecken *, Eric Dufrêne **, Stuart PM RoberTS *** et Jan SMIT ****
}

\begin{abstract}
A collecting trip in the Perpignan-Narbonne area (France) has allowed us to catch several specimens of the cuckoo bee Nomada agrestis FABRICIUS (Hymenoptera, Apidae) and its putative host, Eucera nigrilabris LEPELETIER. This Nomada species has not been found for more than a century in this region of southern France. We also provide a distribution map that integrates all the records of $\mathcal{N}$. agrestis available in both private and public insect collections of Western Europe.
\end{abstract}

Résumé. Au cours de prospections réalisées dans le secteur de Perpignan-Narbonne (France) au cours du printemps 2007, nous avons capturé plusieurs spécimens de l'abeille cleptoparasite Nomada agrestis FABRicius (Hymenoptera, Apidae) et de son hôte probable, Eucera nigrilabris LEPELETIER. Cette espèce de Nomada n'avait plus été capturée depuis plus d'un siècle dans cette région du sud de la France. Nous fournissons également une carte de distribution originale compilant les données biogéographiques de $\mathcal{N}$. agrestis disponibles dans les collections entomologiques de plusieurs institutions privées et publiques d'Europe occidentale.

Mots-clés. Nomada agrestis, Apidae, parasitisme, Eucera nigrilabris.

\section{Les relations hôtes-parasites chez les abeilles}

Le terme parasite est souvent utilisé dans différents contextes, et la littérature contemporaine comporte de nombreuses définitions s'appliquant tant à des organismes animaux que végétaux. S'il existe des différences subtiles entre toutes ces définitions, la grande majorité des auteurs s'accordent cependant sur le fait qu'un parasite est un organisme qui vit au dépend d'un autre organisme (son hote) dont il puise sa nourriture, un abri, ou tout autre élément lui permettant de boucler son cycle biologique (Poulin 2007). Cette définition inclut notamment toute une série de parasites "comportementaux" comme ceux qui exploitent leur(s) hôte(s) par des moyens alternatifs, comme par exemple en exploitant les ressources alimentaires accumulées par leur(s) hôte(s) (Barnard 1990). Le mode de vie parasitaire implique le plus souvent le développement de toute une série d'adaptations spécifiques et parfois également la perte d'autres caractéristiques comportementales/ morphologiques/physiologiques propres aux groupes non-parasites. Chez les abeilles non-parasites (Hymenoptera, Apoidea), les femelles sont dans la plupart des cas équipées d'appareils de récolte de pollen

\footnotetext{
* Eco-Ethologie Evolutive, Université Libre de Bruxelles CP 160/12, Av. F.D. Roosevelt 50, B-1050 Bruxelles, Belgique.

E-mail: nicolas.vereecken@ulb.ac.be

** Laboratoire Ecologie, Systématique et Evolution (ESE), UMR8079 (Université Paris-Sud, CNRS, AgroParisTech), Bât. 362, F-91405 Orsay, France.

E-mail: eric.dufrene@u-psud.fr

*** Centre for Agri-Environmental Research, University of Reading, Earley Gate, PO Box 237, Reading RG6 6AR, United Kingdom. E-mail: s.p.m.roberts@reading.ac.uk

**** Voermanstraat 14, 6921 NP Duiven, Pays-Bas.

E-mail: smit.jan@hetnet.nl
}

ou autres ressources florales sur leurs pattes, leur thorax, leur tête ou leur abdomen. Chez les espèces parasites, les femelles pondent directement dans le nid de leur(s) hôte(s) et on observe presque systématiquement une absence de telles structures morphologiques destinées à la récolte de ressources florales (Westrich 1989; Müller et al. 1997; Michener 2000).

Comme c'est le cas chez de nombreux autres groupes d'organismes (p.ex. Ronquist 1994; Goff et al. 1997), le parasitisme est apparu à plusieurs reprises de façon indépendante au cours de l'évolution des abeilles (voir par exemple Michener 1978, 2000; Bogusch et al. 2006; Danforth et al. 2006; Müller 2006). Les abeilles parasites ont généralement un spectre d'hôtes relativement réduit, le plus souvent moins d'une demidouzaine d'espèces, et les parasites sont régulièrement proches de leurs hôtes d'un point de vue phylogénétique (p.ex. les Sphecodes (Halictidae) parasitent des Halictus et Lasioglossum (Halictidae); les Melecta (Apidae) parasitent des Anthophora (Apidae); les Bombus (Psythirus) (Apidae) parasitent d'autres Bombus (Apidae), etc.).

Ces facteurs importants de l'écologie et de l'évolution des relations parasitaires chez les abeilles intègrent parfaitement le cadre théorique des relations hôtes-parasites, sur lequel il est intéressant de se pencher pour découvrir de nouvelles pistes de recherches pour mieux comprendre la nature et l'évolution de ces relations entre organismes apparentés. A titre d'exemple, Poulin (2007) insiste sur le manque de données disponibles sur la variabilité spatiale et temporelle du spectre d'hôtes de nombreux parasites, ainsi que sur les déterminants et les implications écoéthologiques des relations hôtes-parasites en général. 


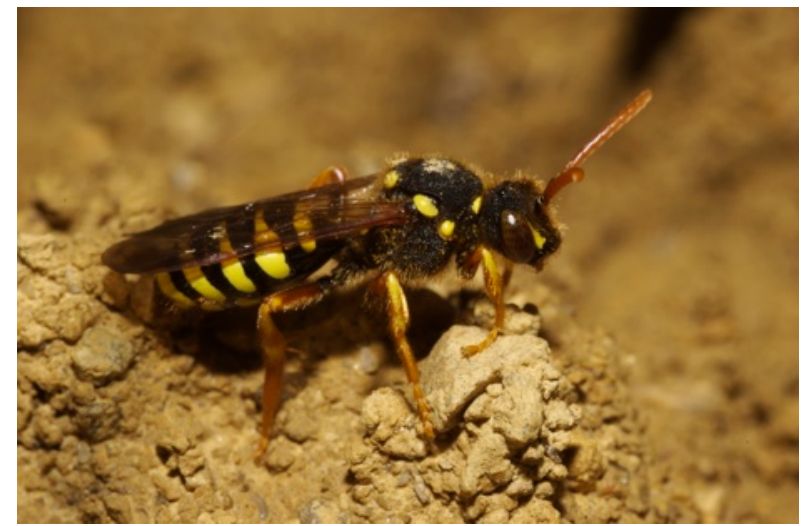

Figure 1. Femelle de Nomada goodeniana (KIRBY) (Hymenoptera, Apidae), 8.III.2007, Cadillon (F) (Photo NJ Vereecken)

\section{Le genre Nomada ScopolI}

Avec leur livrée souvent marquée de jaune, de blanc ou de rouge et leur pilosité éparse et fine, les Nomada font penser à des guêpes (Figure 1). Contrairement à la plupart des lignées d'abeilles cleptoparasites, les Nomada sont représentées par un grand nombre d'espèces, qui comprend à lui seul plus de la moitié des 1200 espèces connues de la sous-famille des Nomadinae (Hymenoptera, Apidae). On trouve des espèces de Nomada sur tous les continents à l'exception de l'Antarctique, la majorité d'entre elles étant présentes dans l'hémisphère nord. L’origine biogéographique du genre reste controversée, Michener (2000) suggère une origine néotropicale sur la base d'un hôte proche d'un point de vue systématique alors qu'Alexander (1994), dans une large étude phylogénique, situe l'origine du genre en Afrique sub-saharienne. Bien que la plupart des Nomada soient des cleptoparasites des espèces du genre Andrena (Andrenidae), quelques-unes parasitent les espèces des genres Panurgus (Andrenidae) et Melitta (Melittidae), mais aussi de la sous-famille des Halictinae (Halictus et Lasioglossum, Halictidae), ou encore du genre Eucera (Apidae) et probablement aussi Colletes (Colletidae) (Smit 1999). Ce large spectre d'hôtes a permis aux Nomada d'atteindre une expansion géographique bien plus large que celle de son hôte principal, le genre Andrena.

Si la faune des Nomada d'Europe occidentale comporte encore d'importantes lacunes, les travaux de

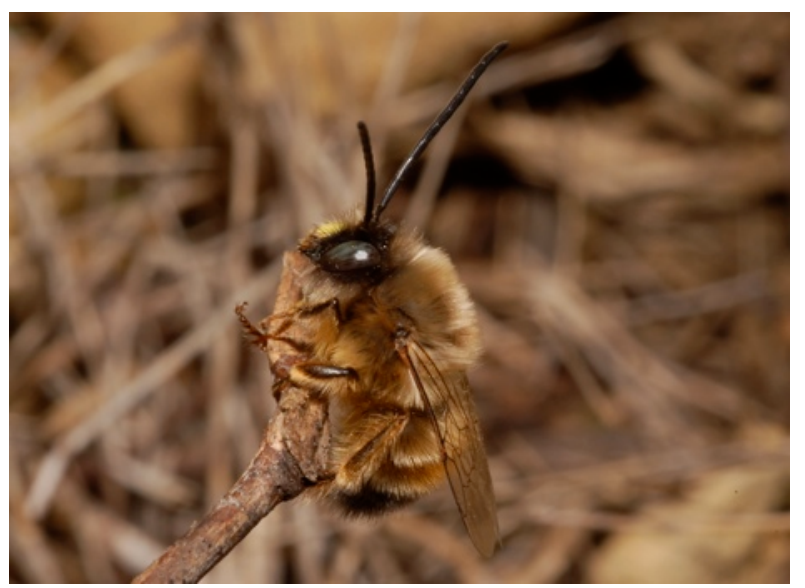

Figure 3. Mâle d'Eucera nigrilabris LEPELETIER (Hymenoptera, Apidae) à l'émergence, 4.III.2007, Narbonne (F) (Photo NJ Vereecken)

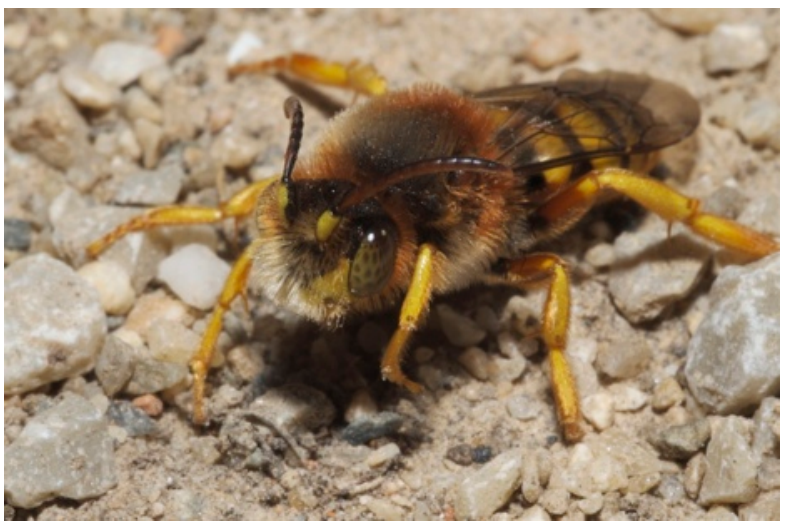

Figure 2. Mâle de Nomada agrestis FABRICIUS (Hymenoptera, Apidae), 11.III.2007, Narbonne (F) (Photo NJ Vereecken)

synthèse (p.ex. Perkins 1919; Stoeckhert 1930; Nobile 1990; Scheuchl 2000; Smit 2004) ont permis de mieux appréhender la diversité et l'écologie de ce groupe d'abeilles sauvages. Cependant, de nombreux aspects de l'écologie des Nomada demeurent obscurs, tels l'amplitude du spectre d'hôtes et la stratégie adoptée par les femelles gravides pour détecter les entrées de nids des espèces-hôtes.

\section{Nomada agrestis FABRICIUS en France}

Au cours de la première quinzaine du mois de mars 2007, nous avons réalisé des prospections dans la région de Perpignan-Narbonne (Aude, France). Nos recherches se sont particulièrement orientées sur différents sites comme le camp militaire de Rivesaltes $\left(42.7944^{\circ} \mathrm{N}\right.$, $2.86719^{\circ} \mathrm{E}$, WGS84) ou encore l'aire de NarbonneJonquières de l'autoroute A61 $\left(43.1545^{\circ} \mathrm{N}, 2.95499^{\circ} \mathrm{E}\right.$, WGS84) où nous avons capturé plusieurs spécimens de chaque sexe de $\mathcal{N}$. agrestis, une abeille cleptoparasite de grande taille (12,5-14,5mm). A l'époque de nos observations, la faune d'abeilles sauvages était relativement peu diversifiée sur les sites d'étude. Nos recensements ont tout de même permis d'inventorier les espèces suivantes sur le site du camp militaire de Rivesaltes : Andrena (Melandrena) nigroaenea (KIRBY) (Andrenidae), Anthidium (Rhodanthidium) sticticum FABRICIUs (Megachilidae), Anthophora (Anthophora) plumipes (PALLAS) (Apidae), Eucera (Pareucera) caspica MORAwitz, E. (Hetereucera) elongatula VACHAL et $E$. (Eucera) nigrilabris LEPELETIER (Apidae) (Figure 3).

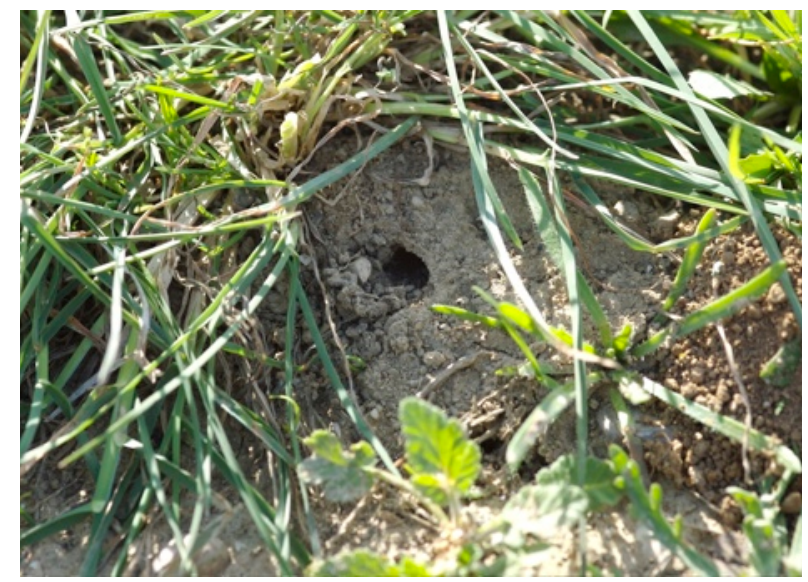

Figure 4. Entrée du nid souterrain d'Eucera nigrilabris LEPELETIER (Hymenoptera, Apidae), 16.III.2007, Narbonne (F) (Photo NJ Vereecken) 


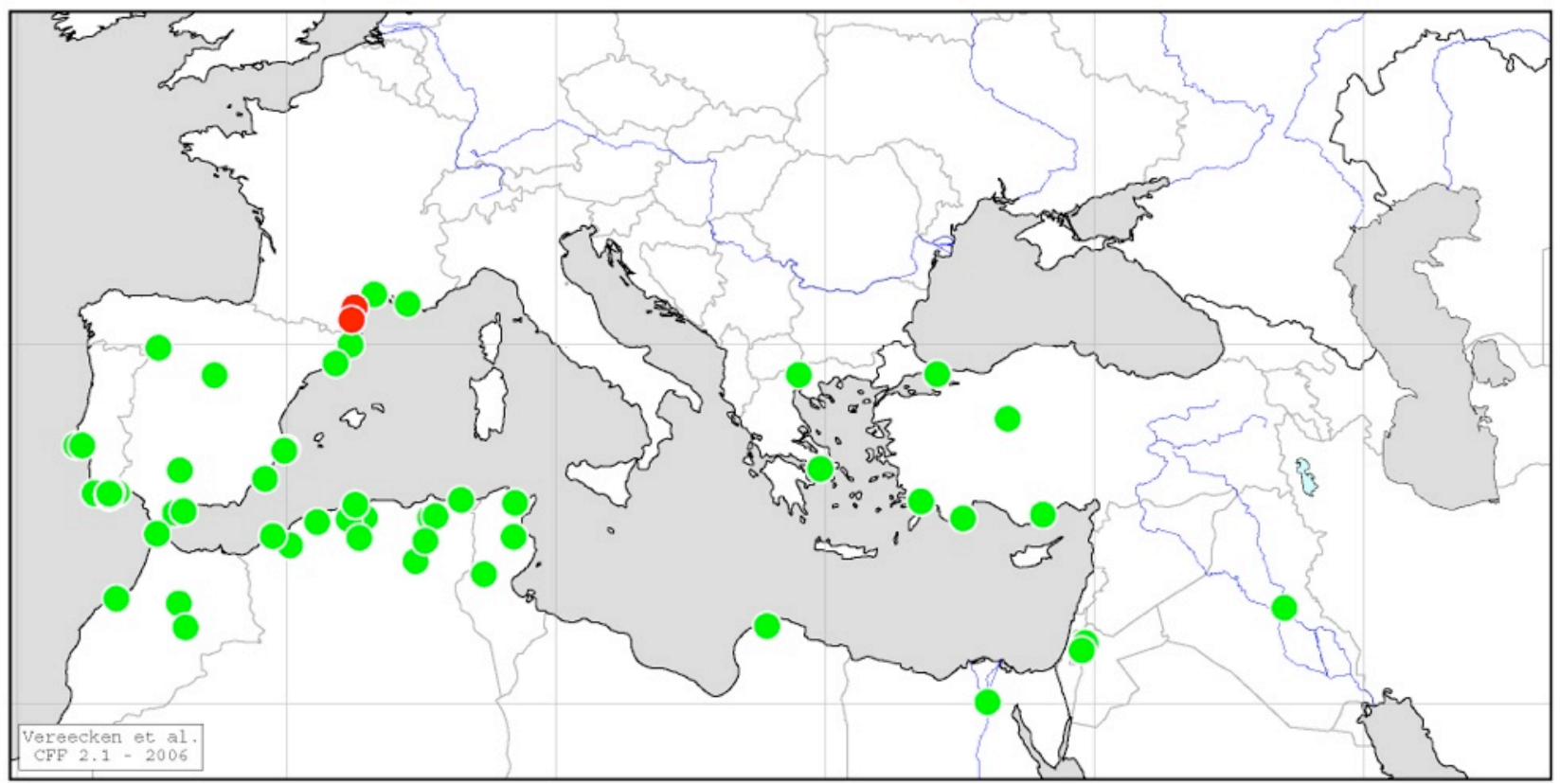

Figure 5. Distribution des observations de Nomada agrestis FABRICIUS (Hymenoptera, Apidae) en Méditerranée. Les points verts indiquent les données antérieures à 2007, les points rouges correspondent aux données de la présente étude (printemps 2007) (Illustration Y. Barbier).

Le site de Rivesaltes englobe une vaste étendue de plusieurs hectares de garrigue où poussent de nombreuses espèces végétales telles Asparagus acutifolius L. (Liliaceae), Calycotome spinosa (L.) (Fabaceae), Euphorbia characias L. (Euphorbiaceae), Juniperus oxycedrus L. (Cupressaceae), Muscari neglectum Guss. (Liliaceae), Olea europaea L. (Oleaceae), Ophrys arachnitiformis GRENIER \& PhiliPPe, O. bilunulata RISSO, O. lupercalis DevillersTerschuren \& Devillers, Phillyrea angustifolia L. (Oleaceae), Rosmarinus officinalis L., ou encore Thymus vulgaris L. (Lamiaceae) (Voir Vereecken et al. 2007). Le site de Narbonne-Jonquières, quant à lui, constitue un endroit a priori peu accueillant pour l'apidofaune puisqu'il s'agit d'une aire d'autoroute isolée, largement macadamisée, et dont les espaces verts semi-naturels se limitent à quelques talus et pelouses où poussaient entre autres Diplotaxis erucoides (L.) (Brassicaceae), Ophrys arachnitiformis GRENIER \& PHILIPPE (Orchidaceae), Phillyrea angustifolia L. (Oleaceae), Rosmarinus officinalis L. et Thymus vulgaris L. (Lamiaceae). Outre plusieurs spécimens de $\mathcal{N}$. agrestis, les seuls Apoïdes recensés sur ce site entre le 4 et le 18 mars 2007 sont les suivants : Andrena (Andrena) fulva (MÜLLER), Andrena (Biareolina) lagopus LATREILLE (Andrenidae) ainsi qu'Eucera (Eucera) nigrilabris LEPELETIER (Apidae). Des entrées de nids occupés de cette dernière espèce ont notamment pu être observées (Figure 4) et plusieurs spécimens ont été capturés pour identification.

Au cours des mois qui ont suivi ces observations et la confirmation de l'identification de nos spécimens de $\mathcal{N}$. agrestis, nous avons entrepris de réaliser une compilation des données biogéographiques relatives à cette espèce. Un total de 110 données ont pu être réunies suite à l'examen de différentes collections conservées dans les institutions suivantes : Museum National d'Histoire Naturelle de Paris (MNHN, France), Natural History Museum of London (NHM, Royaume-Uni), Rijksmuseum van Natuurlijke Historie de Leiden
(RNHL, Pays-Bas), Zoologisch Museum d'Amsterdam (ZMA, Pays-Bas), ainsi que dans les collections privées de Jan Smit (Duiven, Pays-Bas) et Stuart PM Roberts (Salisbury, Royaume-Uni). Ces données nous ont permis de créer une carte de distribution de l'espèce (Figure 5) et de constater que, bien que l'espèce semble ouestméditerranéenne comme indiqué par Nobile (1990), plusieurs spécimens ont également été capturés dans la partie orientale du bassin méditerranéen, notamment en Turquie, en Egypte et dans certaines régions de Jordanie et d'Irak (Figure 5).

Les données françaises de $\mathcal{N}$. agrestis concernent des spécimens capturés dans les Bouches-du-Rhône, dans le Var et dans la région de Montpellier. Nous avons également retrouvé deux spécimens (un mâle et une femelle) collectés à Narbonne, dans l'Aude, et conservés dans la collection Saunders du NHM de Londres, et dont l'étiquette indique la date du 7 avril 1903. Tout indique donc que $\mathcal{N}$. agrestis était déjà présente dans le secteur de Narbonne-Perpignan au début du $\mathrm{XX}^{\mathrm{ème}}$ siècle, et que l'espèce n'avait plus été trouvée dans la région depuis plus d'un siècle.

\section{L'hôte de $\boldsymbol{N}$. agrestis en Méditerranée}

Au terme de nos prospections, nous avons constaté qu'E. nigrilabris était la seule espèce commune aux deux sites d'étude où les spécimens de $\mathcal{N}$. agrestis ont été observés et capturés. Ce phénomène à lui seul ne constitue en rien une preuve du lien entre $\mathcal{N}$. agrestis et E. nigrilabris, mais l'hypothèse qu'E. nigrilabris pourrait bien être l'hôte de cette $\mathcal{N}$ omada coïncide bien avec les informations présentées par Friese (1923) qui cite $E$. nigrilabris comme hôte pour $\mathcal{N}$. agrestis, ainsi que Nobile (1990) qui cite Andrena thoracica et "Andrena" (sic) nigrilabris comme hôtes dans les régions circumméditerranéennes. Tout indique donc que nos captures, en particulier celle de $\mathcal{N}$. agrestis et d'E. nigrilabris sur les mêmes sites au 
cours de nos prospections, s'expliquent par les relations qu'entretiennent ces espèces dont les cycles biologiques sont intimement liés.

\section{Remerciements}

Nous remercions chaleureusement Stephan Risch (Leverkusen, Allemagne) qui a eu la gentillesse d'identifier les spécimens d'Eucera capturés au cours de cette étude, ainsi qu'Yvan Barbier (Gembloux, Belgique) qui nous a aimablement aidé à illustrer la distribution des observations relatives à Nomada agrestis en Méditerranée. MM. Andreas Müller (ETH Zürich, Suisse) et Maximilian Schwarz (Ansfelden, Autriche) nous ont aimablement apporté des informations complémentaires au cours de la préparation de cet article, et tous nos remerciements s'adressent également aux curateurs des collections entomologiques étudiées, en particulier D. Notton au NHM de Londres.

\section{Références bibliographiques}

Barnard CJ, 1990. Parasitic relationships. In CJ Barnard \& JM Behnke (eds.), Parasitism and host behaviour, pp. 1-33, Taylor \& Francis, London.

Bogusch P, Kratochvil L \& Straka J, 2006. Generalist cuckoo bees (Hymenoptera: Apoidea: Sphecodes) are species-specialist at the individual level. Behavioral Ecology and Sociobiology 60: 422-429.

Danforth BN, Sipes SD, FangJ \& Brady SG, 2006. The history of early bee diversification based on five genes plus morphology. Proceedings of the National Academy of Sciences of the United States of America 103: 15118-15123.

Else GR, (en prépation). The Bees of the British Isles.

Goff LJ, Ashen J \& Moon D, 1997. The evolution of parasites from their hosts: a case study in the parasitic red algae. Evolution 51: 1068-1078.

Michener CD, 1978. The parasitic groups of Halictidae (Hymenoptera, Apoidea). Univ Kans Sci Bull 51: 291-339.

Michener CD, 2000. The Bees of the World. The Johns Hopkins University Press, Baltimore, 913p.
Müller A, Krebs A \& Amiet F, 1997. Bienen, Mitteleuropäische Gattungen, Lebensweise, Beobachtung. Natur Buch Verlag, Augsburg, 384 p.

Müller A, 2006. A scientific note on Bombus inexspectatus (TKALCU, 1963): evidence for a social parasitic mode of life. Apidologie 37: 408-409.

Nobile V, 1990. Contributo alla conocenza delle api parassite (Insecta, Hymenoptera) di Sicilia. II. Il genere Nomada SCOPOLI 1770, con descrizione di una nuova specie. Animalia Catania 17: 219-243.

Peeters TMJ, Raemakers IP \& Smit J, 1999. Voorlopige atlas van de Nederlandse bijen (Apidae). European Invertebrate Survey Nederland, Leiden.

Perkins RGL, 1919. The British species of Andrena and Nomada. Transactions of the Entomological Society of London 1919: 218-319.

Poulin R, 2007. Evolutionary ecology of parasites. Princeton University Press, Princeton \& Oxford.

Ronquist F, 1994. Evolution of parasitism among closely related species: phylogenetic relationships and the origin of inquilinism in gall wasps (Hymenoptera, Cynipidae). Evolution 48: 241-166.

Scheuchl, E. 2000. Illustrierte Bestimmungstabellen der Wildbienen Deutschlands und Österreichs. Band i: Anthophoridae. 2. erweiterte Auflage. - eigen uitgave, 158p.

Smit J, 2004. De wespbijen (Nomada) van Nederland (Hymenoptera: Apidae). Nederlandse Faunistische Mededelingen 20.

Stoeckhert E, 1930. Nomada F. - In: Schmiedeknecht, O. (red.), Die Hymenopteren Mitteleuropas (2. Aufl.), Jena: 986-1053.

Tierney SM, Smith JA, Chenoweth L, Schwarz MP, 2008. Phylogenetics of allodapine bees: a review of social evolution, parasitism and biogeography. Apidologie (in press)

Vereecken NJ, Risch S \& Cortis $\mathbf{P}, \mathbf{2 0 0 7}$. A contribution to the pollination biology of Ophrys scolopax CAVANILLES (Orchidaceae) in southern France. Natural. Belges 88 (Orchid. 20): 17-26.

Westrich P, 1989. Die Wildbienen Baden-Wûrttembergs. Spezieller Teil: Die Gattungen und Arten. Eugen Ulmer GmbH \& Co, Stuttgart, 972p.

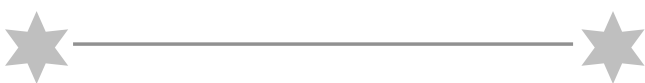

